\title{
The Prefrontal Cortex in a Pandemic: Restoring Functions with System-, Family-, and Individual-Focused Interventions
}

${ }^{1}$ Child Study Center, Yale School of Medicine, New Haven, Connecticut

${ }^{5}$ Department of Psychology, Yale University

${ }^{6}$ Division of Psychology and Language Sciences, University College London

${ }^{7}$ Anna Freud National Centre for Children and Families, London, England

\begin{abstract}
The COVID-19 pandemic is an unanticipated and uncontrollable chronic stressor that is detrimental to the mental and behavioral health of children and families, particularly those from disadvantaged and marginalized backgrounds. Chronic stress impairs a myriad of prefrontal cortical functions, important for coping with the COVID-19 pandemic, and has consequences on dyadic parent-child functioning. Informed by neuroscience and clinical evidence, sensitive parenting is a vital avenue of intervention that buffers against the toxic effects of COVID-19 on parent-child mental health. In the context of the COVID-19 pandemic, we first discuss the neurobiological, psychological, and behavioral mechanisms behind exacerbated mental health risks in families. We then highlight the role of sensitive parenting as a buffer against stressrelated mental health problems, and conclude with recommendations for systemic-, family-, and individual-interventions to most effectively address stress-related mental health problems and their impact on children and families during the COVID-19 pandemic.
\end{abstract}

\section{Public Significance Statement}

This review examines the neurobiological mechanisms underlying exacerbated mental health risks in disadvantaged and marginalized families during the COVID-19 pandemic, and potential interventions. The chronic stress of COVID-19 impacts various prefrontal cortical functions that are critical for adaptive emotion regulation, mentalizing, and coping skills in children and their caregivers. These functions can be restored by systemic policies that address long-standing structural racism and mental health inequities, as well as family- and individual-level interventions that aim to reduce stress and promote sensitive caregiving.

Keywords: COVID-19, developmental psychopathology, prefrontal cortex, sensitive caregiving, toxic stress

Eileen M. Condon (D) https://orcid.org/0000-0001-7747-5870 Amanda M. Dettmer (D) https://orcid.org/0000-0003-1360-3567

Dylan G. Gee (D) https://orcid.org/0000-0002-3685-2710

Ka Shu Lee (D) https://orcid.org/0000-0002-5532-6222

Carla S. Stover (D) https://orcid.org/0000-09 2 _767-5307

Wan-Ling Tseng (D) https://orcid.org/0000-_-8441-7118

Yale Early Stress Atrersity Consortium Ka Shu Lee is now at the Department of Experimental Psychology, University of Oxford.

Yale University and AFTA receive royalties from Shire/Takeda for the USA sales of Intuniv. They do not receive royalties from non-USA or generic sales. The other authors declare no competing financial or nonfinancial interests.
All authors contributed equally to the work. Amy F. T. Arnsten, Eileen M. Condon, and Amanda M. Dettmer designed the figure. Eileen M. Condon is supported by the National Institute of Nursing Research of the National Institutes of Health (K99NR018876). Amanda M. Dettmer is supported by a Clinical Science and Translational Award from the National Center for Advancing Translational Sciences (UL1TR001863). Wan-Ling Tseng is supported by a research Grant from the National Institute of Mental Health (R00MH110570). We gratefully acknowledge M. Stover for her assistance in developing the figure.

Correspondence concerning this article should be addressed to Ka Shu Lee, Yale Child Study Center, 230 South. Frontage Road, New Haven, CT 06519, United States. Email: kashu.lee@yale.edu 


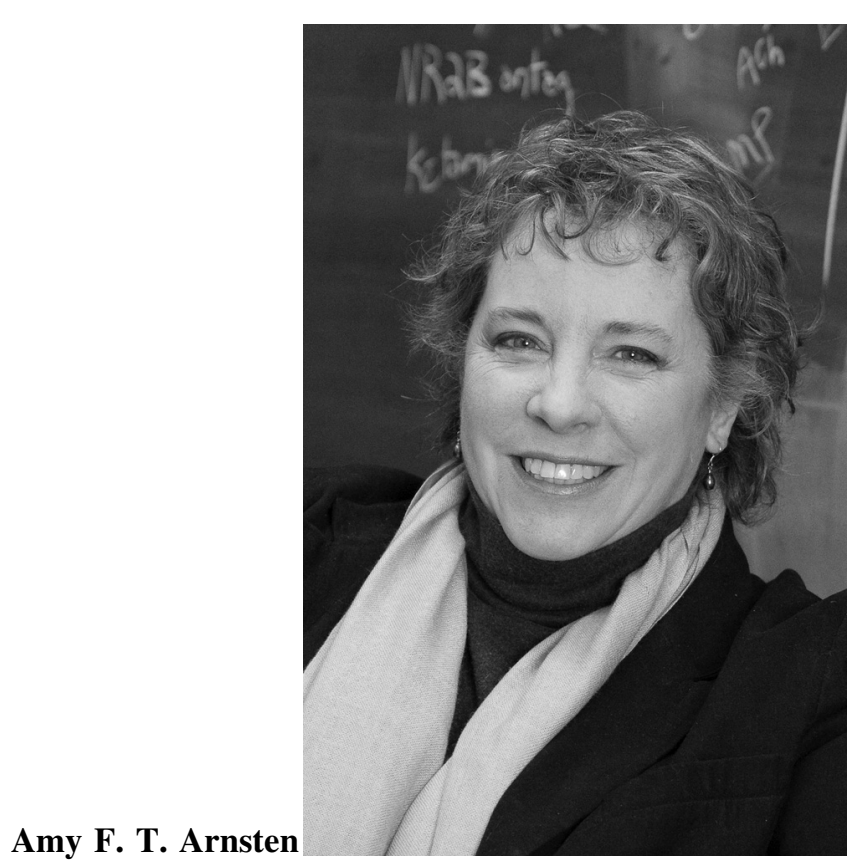

Amy F. T. Arnsten

The COVID-19 pandemic is a major stressor that is disrupting family functioning and placing family members of all ages at risk for behavioral and mental health disorders. Children have had to adapt abruptly to school closures and the loss of predictable daily routines therein. Parents and caregivers are simultaneously juggling the roles of homeschool teachers and caregivers while maintaining occupational responsibilities. Further, the protections required to safeguard against COVID-19 infection also block access to activities and social relationships that are known to reduce the effects of stress (Gruber et al., 2020). In addition, the national unrest surrounding anti-Black racism in the United States brings attention to pervasive health inequities: COVID-19, the illness caused by the severe acute respiratory syndrome coronavirus 2 (SARS-CoV-2), is exacerbated for many due to preexisting chronic stressors stemming from systemic racism (Condon et al., 2020). Thus, considering the chronicity and unprecedented scale of the COVID-19 pandemic, children and families are in critical need of effective mental health care.

Disruptions in routine and loss of resources related to the COVID-19 pandemic are particularly harmful for children living in families that experienced prepandemic inequities and disadvantages including lower economic resources, reduced access to health care, lower-quality education, and myriad other adversities stemming from systemic racism and inequities in the U.S (Condon et al., 2020). For these families, the pandemic has exacerbated preexisting inequities (Condon et al., 2020). At least an estimated 44 to 66 million more disadvantaged children and families worldwide have experienced at least one form of socioeconomic disparity since the COVID-19 pandemic began, including systemic poverty, an academic achievement gap, malnutrition due to food insecurity, job loss, and the threat of homelessness in discriminatory, unsafe neighborhoods (United Nations, 2020). Children who relied upon schools for food security, mental health support, and important social interactions may lack the resources to access and maintain these supports during the pandemic. Similarly, their caregivers are likely facing job, food, housing, and health care insecurity, resulting in forced choices between work and childcare (Condon et al., 2020). Many such caregivers are low-wage frontline workers who are also more likely to experience difficulty with enacting physical distancing at home, thereby increasing stress and the potential for COVID-19 infection. Consequently, caregivers are at increased risk for anxiety, depression, and substance misuse, and their children are at increased risks for neglect, maltreatment, family violence, and mental health symptoms and difficulties (Condon et al., 2020; Humphreys et al., 2020).

Due to this heightened duress, all families, particularly those from disadvantaged or marginalized communities, are at increased risk for uncontrollable stress. Chronic and uncontrollable stress can stem from societal factors such as lack of access to quality education, employment, and health care, all of which are needed for individuals to thrive. These types of stress involve repeated or sustained activation of the biological stress system, such as occurs with the COVID-19 pandemic, and over time leads to physical, behavioral, and mental health problems (McEwen \& McEwen, 2017). Encouragingly, even in the presence of significant adversities, a chronic stress response can be buffered when children experience a sense of control through secure attachment relationships and sensitive caregiving, defined by persistent quality connections consisting of caregiver responsivity and "serve and return" interactions (Levitt \& Eagleson, 2018). However, the myriad stressors stemming from the COVID-19 pandemic are likely to strain caregivers' abilities to provide sensitive caregiving, and caregivers with histories of adversity are particularly at risk (Condon et al., 2020). Thus, without adequate support, pandemic-related stressors are certain to have a significant impact on caregiver-child relationships and child mental health, and may further exacerbate preexisting inequities.

This review leverages knowledge from neuroscience to describe how pandemic-related stressors may affect the brain, and based on that knowledge, recommends intervention strategies to help mitigate potentially harmful effects on caregiver-child relationships and child mental health. We describe mechanisms through which stress impacts the brain, particularly the prefrontal cortex, and subsequent effects on behavior. The importance of this understanding is threefold: (a) both children's and adult caregivers' brains 


\section{Eileen M. Condon}

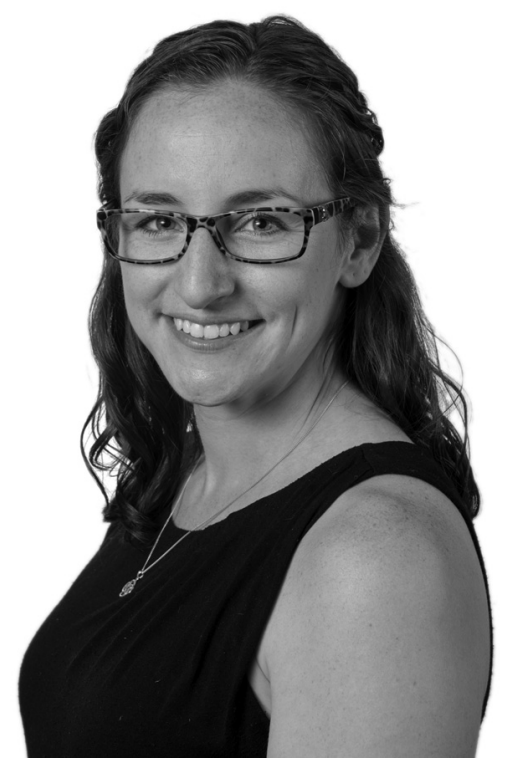

may be affected by the stress of the COVID-19 pandemic, placing them at increased risk for poor mental health outcomes; (b) pandemic-related stressors may impair caregivers' ability to provide sensitive caregiving and buffer chronic stress for their child; and (c) impairments in caregiving may be exacerbated among caregivers with a prior history of stress and adversity. Informed by these studies on the neurobiological and psychological mechanisms related to stress responses and family-level factors, we then describe policy-, family-, and individual-level interventions to help alleviate family stress and promote healthy caregiver-child relationships simultaneously, and advocate for a multilevel approach to effectively support all children and families through the COVID-19 pandemic.

\section{Mechanisms of Stress-Related Mental Health Problems}

Stress is a known predictor of mental health disorders (e. 5., Breslau, 2009). A stressor that is unpredictable and uncontrollable such as the COVID-19 pandemic (e.g., dealing with an invisible, pervasive virus where no place may feel safe while vaccine development is still underway) can have a profound effect on individuals' brain function. The effect on the developing brain may be particularly pronounced (Cohodes et al., 2020); putting children and adolescents at heightened risk for mental health problems and possibly hindering their ability to cope with future stressors following the pandemic. Indeed, research in rodents, monkeys, and humans has shown that exposure to uncontrollable stress rapidly impairs the functioning of the prefrontal cortex (PFC), a newly evolved brain region needed to maintain a healthy mental state (Arnsten, 2009; Liston et al., 2006).

\section{Impaired PFC Functions Under Stress}

Prefrontal regions subserve higher cognitive functions that are important to guide flexible, goal-directed behavior, and top-down regulation of emotion, attention and action. Prefrontal circuitry has the extraordinary ability to generate mental representations in the absence of sensory stimulation, the foundation of abstract thought. Thus, the PFC is especially important in dealing with an invisible virus (e.g., using abstract reasoning to imagine the potential harm in once habitual behaviors like hugging a friend). Prefrontal circuitry is also needed for effective, sensitive parenting, as it supports the mentalizing functions that allow a parent to understand their child's state of mind in response to pandemic-related challenges, such as disruptions in family routines, and to plan effective strategies to help children cope with restrictions in learning and socializing with peers. The PFC is also part of the broader neural circuitry that mediates attachment and empathy as well as self-regulation, such as inhibiting irritable and angry outbursts and enabling selfsoothing, both of which are vital emotion regulation skills to cope with frustration caused by disruptions in work and social lives such as those caused by the pandemic. The PFC accomplishes these multiple functions through its extensive connections throughout the brain, and can inhibit the actions of more primordial brain structures, such as the amygdala and basal ganglia, which are involved in reactive, emotional responses like shouting and aggression.

The PFC accomplishes high order functions through recurrent excitatory circuits, where PFC neurons activate each other through glutamatergic, NMDA receptor connections to maintain information in mind. A recent study found increased levels of kynurenic acid (KYNA) in plasma after COVID-19 infection (particularly in men) (Cai et al., 2020). Given that KYNA blocks NMDA receptors, this could otentially weaken functions such as working memory and hhibitory control that depend on the PFC. Likewise, high levels of catecholamines norepinephrine (NE) and dopamine (DA) are released in the brain upon exposure to uncontrollable stressors like COVID-19, weakening the aforementioned connections (Arnsten, 2015). In particular, high levels of catecholamine released in the PFC rapidly weaken connections by opening potassium channels near PFC synapses, which impairs higher cognitive functions and top-down control. In contrast, high levels of catecholamines in the amygdala and basal ganglia simultaneously strengthen the more primitive, emotional responses subserved by these brain regions, shifting the brain from a more reflective to a more reflexive state (Arnsten, 2015), which could be maladaptive when coping with the rapidly evolving pandemic. 


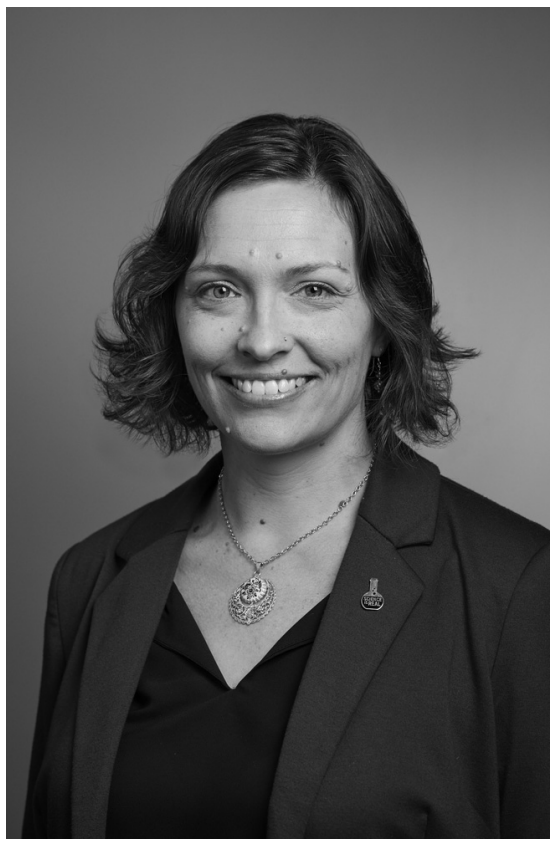

Amanda $M$. Dettmer

In rodents, with prolonged stress exposure, there is actual loss of dendrites and spines in the PFC (e.g., Liston et al., 2006; Ota et al., 2014); which correlates negatively with measures of working memory (Hains et al., 2009) and attention shifting (Liston et al., 2006). In contrast, chronic stress increases dendritic growth in the amygdala (Vyas et al., 2002); strengthening circuits involved in emotional reactivity. In humans, chronic stress is associated with reduced PFC functional connectivity and attention control in adults (Liston et al., 2009); and childhood poverty and chronic stress are associated with dysregulated prefrontal control of the amygdala in adulthood (Kim et al., 2013). In contrast, increased volume in the amygdala in children and adolescents has been found following toxic stress (e.g., maternal depression and childhood maltreatment; Lupien et al., 2011; Whittle et al., 2013). These data further corroborate the idea that stress exposure, acute or chronic, impairs higher PFC cognitive functions and strengthens amygdala-striatal affective responding (Arnsten, 2015). Disruption of higher order PFC-mediated functions during and following the COVID19 pandemic may manifest psychologically and behaviorally as heightened emotional reactivity (e.g., hypervigilance to threat), blunted reward responsivity, emotion dysregulation, and weaker mentalizing (Arnsten, 2015; Duffy et al., 2018; Germine et al., 2015). On a positive note, animal research shows that the dendritic loss can be reversed at a young age after spending an adequate amount of time under safe conditions (Bloss et al., 2011). That is, neural plasticity, be it fostered by sensitive caregiving or multilevel interventions as discussed later in this paper, can be leveraged to rescue compromised PFC functions following stress, such as the current pandemic. For a more detailed depiction of the effects of stress on the brain, see https://youtu.be/ TsQUeNuvIDY.

\section{Heightened Emotional Reactivity}

Adversities such as neglect, physical abuse, and exposure to domestic violence, are likely magnified due to increased family stress caused by the COVID-19 pandemic (Condon et al., 2020). Evidence suggests that children who experience these adversities show heightened emotional reactivity (e.g., quicker and biased responses to identify angry faces with less perceptual information), lower threshold for (mis) classifying emotional faces as threatening, and greater difficulty disengaging from threat stimuli (e.g., Briggs-Gowan et al., 2015; Pollak \& Tolley-Schell, 2003). Such heightened emotional reactivity is mediated by increased amygdala reactivity to threat (Hein \& Monk, 2017); which in turn can lead to greater perceived stress in both adults and children (McLaughlin et al., 2010), creating a snowballing effect in the demands to cope with COVID-19 alongside existing adversities and systemic inequities.

\section{Blunted Reward Responsivity}

Emerging studies have confirmed that food insecurity has increased during the pandemic (Adams et al., 2020). Especially among families that inherently struggle with nutritious food supply, stockpiling shelf-stable foods as a strategy to cope with heightened food insecurity has been associated with increased urgency/need to eat, increased consumption of ultraprocessed snacks, and heightened concern about children's weight when children had more sedentary time at home following previous school closures (Adams et al., 2020). These data may reflect instances of reinforced overeating (a maladaptive reward-seeking behavior) in response to pandemic-related stress, putting marginalized children at substantial risk for physical and brain health issues (e.g., overweight/obesity) (Rundle et al., 2020). Indeed, there is evidence that food insecurity is associated with poorer performance on a reward processing task and reduced white matter integrity in frontostriatal tracts, conferring increased risk for depression (Dennison et al., 2019). Likewise, child neglect and caregiver deprivation, both of which are exacerbated during the pandemic as aforementioned, are associated with blunted reward responsivity mediated by reduced ventral striatum reactivity (Hanson et al., 2015; 2016), which has been found to induce reward-seeking behavior (Blum et al., 2000) including substance use (Duffy et al., 2018). 


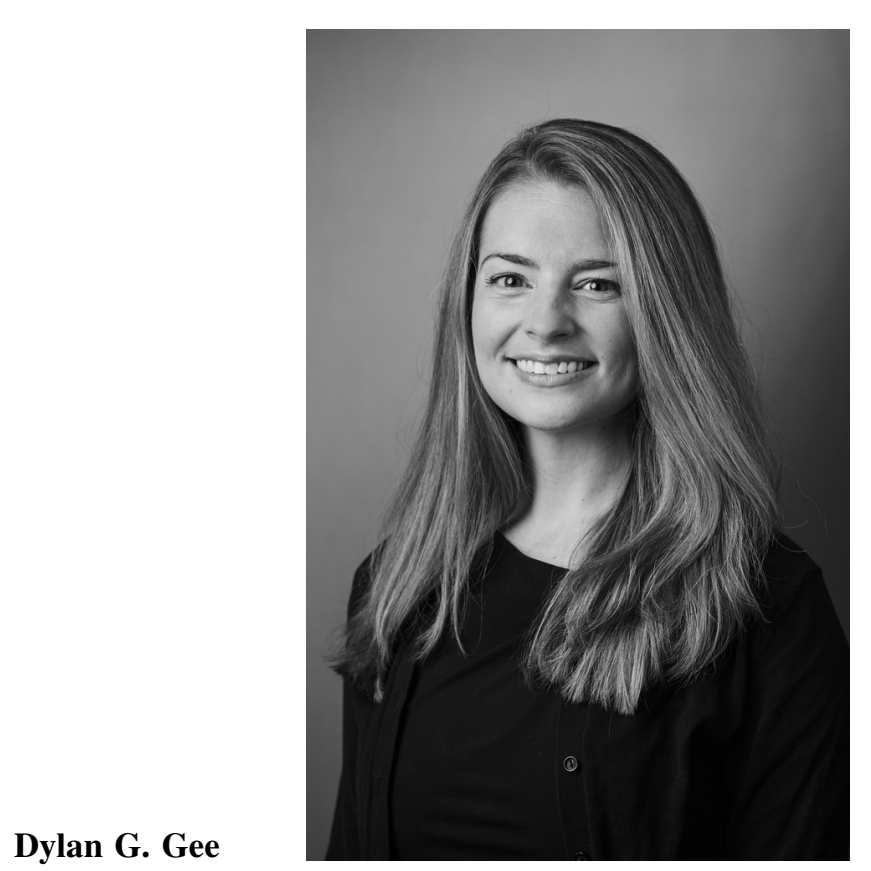

\section{Emotion Dysregulation}

One of the most profound effects of severe stress related to the pandemic is emotion dysregulation mediated by alterations in the PFC-amygdala circuitry (Duffy et al., 2018; Kim \& Cicchetti, 2010). Adolescents exposed to child maltreatment show elevated emotional reactivity and greater habitual use of maladaptive responses to cope with distress (e.g., rumination and impulsive and sensation-seeking behaviors; Heleniak et al., 2016). Relative to nonmaltreated peers, maltreated children show fewer adaptive emotion regulation skills and receive less maternal validation and emotion coaching when communicating emotionally arousing scenarios with their mothers (Shipman et al., 2007). Emotion dysregulation also relates to increased likelihood of primordial, reflexive responding to threat, in particular higher levels of aggression (Park et al., 2018). Weaker resting state connectivity in the PFC-amygdala circuitry is related to increased exposure to significant stressful life events (e.g., sudden absence or death of a primary caregiver due to COVID-19) and higher levels of frustration and aggression in children (Park et al., 2018); which could arise due to a prolonged need for self-control in the context of home confinement, social distancing, and mask wearing during the pandemic. Long-term emotion dysregulation increases the risk of both internalizing and externalizing psychopathology in youths, notably anxiety and mood disorders following adversities (Duffy et al., 2018; Heleniak et al., 2016) including the COVID-19 pandemic.

\section{Maladaptive Mentalizing}

Early adversities, especially childhood maltreatment and trauma which are likely to be exacerbated during the pandemic, hinder mentalizing development in youths (Germine et al., 2015). This scaffolding is mediated by the PFC-temporoparietal circuits. Mentalizing refers to the ability to comprehend and reflect on the mental states of oneself and others, notably feelings, intentions, and wants (Fonagy \& Bateman, 2016). Adaptive mentalizing depends largely on long-term and quality parent-child coupling, which is crucial to future interpersonal functioning. Recent estimates have projected that every COVID-19 death in the United States will leave approximately 9 bereaved relatives (with more disproportionate effects among Black, Latino, and Indigenous populations; Verdery et al., 2020). The absence of an attachment figure to mentalize and communicate with can be particularly detrimental to the socioemotional wellbeing of vulnerable children. Neuroscience and behavioral studies have shown that chronic exposure to stress in early development (as many are experiencing during the COVID19 pandemic) causes a shift from controlled mentalizing (a reflective state) to less sensitive or biased automatic inference of socioaffective signals (a reflexive state), which often leads to low social motivation and ostracism (Fonagy \& Bateman, 2016). Prolonged social restrictions and uncertainty not only further reinforce the reflexive state of mind that "interpersonal relationships are not as rewarding" but also perpetuate a vicious cycle between low social motivation and social isolation in marginalized families, both of which are robust predictors of low self-esteem and elevated risk for anxiety and depression (Germine et al., 2015; Luyten et al., 2020). Indeed, maladaptive mentalizing is considered a transdiagnostic factor (Luyten et al., 2020), suggesting a potential focal point for intervention during and following the COVID-19 pandemic.

\section{Timing and Developmental Considerations}

Recent translational research has suggested that the timing of stress (i.e., age onset and duration) is especially relevant to its neurobiological consequences and associated mental health risks across the life span (Gee \& Casey, 2015). Connections between the PFC and amygdala undergo dynamic changes across development (Gee et al., 2013; Silvers et al., 2017). In addition, the buffering effect of caregivers on children's stress reactivity may vary across developmental stages. For example, one study found that maternal presence (a caregiver's image) dampened amygdala reactivity in children (4 to 10y) but not adolescents (11 to $17 \mathrm{y}$ ), suggesting that caregivers play a unique role in buffering stress during this period of socioemotional development (Gee et al., 2013), which is also a developmental period that is stressful for parents. Parenting stress may be especially heightened among disadvantaged families that 


\section{Ka Shu Lee}

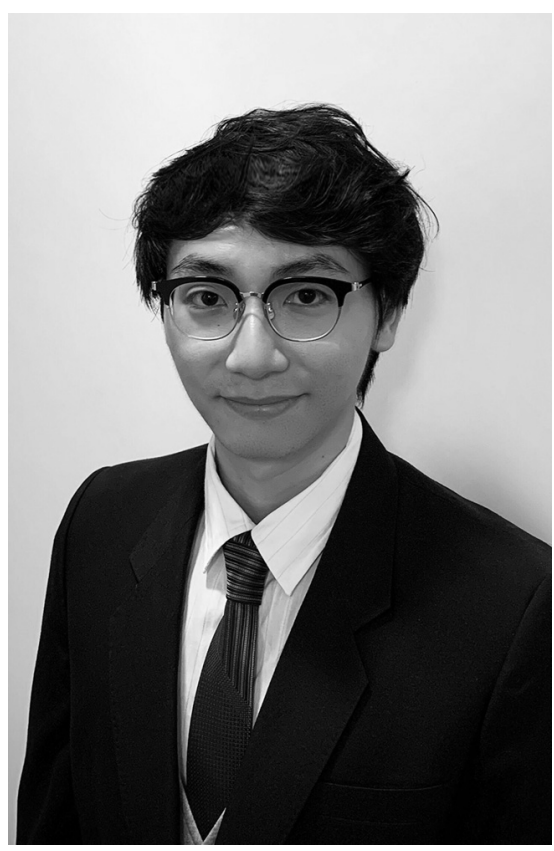

reported a loss of regular childcare, insurance status, and worsening food and job security during the pandemic (Patrick et al., 2020). In contrast, adolescence is characterized by increasing independent regulation and sociocultural learning via peer relationships. Yet the salience of peer relationships also increases adolescents' susceptibility to loneliness due to social isolation during the pandemic (Loades et al., 2020). Such heightened sense of loneliness in adolescents can be of particular concern among marginalized families where parents cannot afford to work from home and have less time for quality parent-child interactions because of the forced choice to prioritize job security and breadwinning in times of a pandemic (Orben et al., 2020). Together, it is important to consider the differential effects of COVID-19 pandemic on individual developmental periods with distinct patterns of prefrontal-amygdala circuitry, developmental tasks/demands, and stress buffering. While mental health professionals may tailor policy recommendations based on the specific needs of marginalized youths across developmental periods, tailoring interventions based on developmental stage and the biological state of the developing brain is a promising approach to be taken in concert so as to optimize the intervention efficacy across multiple levels (Lee et al., 2014).

\section{Buffering Effect and Neural Basis of Sensitive Caregiving}

Caregivers play a central role in buffering stress early in life. How caregivers respond to children's stress and convey information about traumatic events has critical effects on children's mental health following stress (Carpenter et al., 2017). Neuroscience research across species has elucidated the neurobiological mechanisms by which caregivers may buffer stress reactivity in children, findings which have directa implications for caregivers during the pandemic and beyond. In rodents, maternal presence maintains low levels of corticosterone and blocks amygdala plasticity (Moriceau \& Sullivan, 2006). Consistent with these findings, caregiver presence buffers hypothalamic-pituitary-adrenal (HPA) axis reactivity in infant macaques (Sanchez, 2006) and suppresses cortisol reactivity in children (Kertes et al., 2009).

Evidence in humans has shown that parental cues dampen amygdala reactivity in childhood (Gee et al., 2013). Children also display a more mature pattern of frontoamygdala connectivity to their caregiver's face, suggesting that greater prefrontal control may support these effects of parental buffering. Paralleling these neural findings, behaviorally, children perform with better regulatory control in an affective context when in the physical presence of their parent versus a stranger. Together, these findings highlight the important role that parents and caregivers play in supporting children's brain function and regulatory behavior, particularly under stressful situations such as the COVID-19 pandemic.

Importantly, evidence suggests that disruptions in caregiving and parenting stress both can interfere with the buffering effects of caregivers on children's neural and behavioral emotional reactivity. Across species, maltreatment during infancy reduces the buffering effects of caregiving (rodents: Opendak et al., 2019; nonhuman primates: Sanchez et al., 2015; humans: Callaghan et al., 2019). The extent to which parents are stressed also influences the ways in which they regulate children's stress and emotions. Parenting stress and family stress, which are at heightened during the pandemic (Brown et al., 2020; Cluver et al., 2020); have been shown to positively and longitudinally predict suboptimal relational functioning with children and negative parenting behaviors, exacerbating the mental health impact of adversities (Palmer et al., 2013).

Caregiving and parenting stress as a result of adversities and related parental psychopathologies also impair the mentalizing capacity of caregivers to comprehend and respond contingently to children's socioemotional needs. In postpartum mothers, prefrontal regions were important for parental mentalizing (e.g., dorsal anterior cingulate cortex) and were most strongly activated when viewing synchronized mother-infant interactions but not mismatched interactions between infants and mothers diagnosed with postpartum depression and anxiety (Atzil et al., 2014). Under adverse circumstances like those exacerbated by the pandemic, children are more prone to a state of mind characterized by 


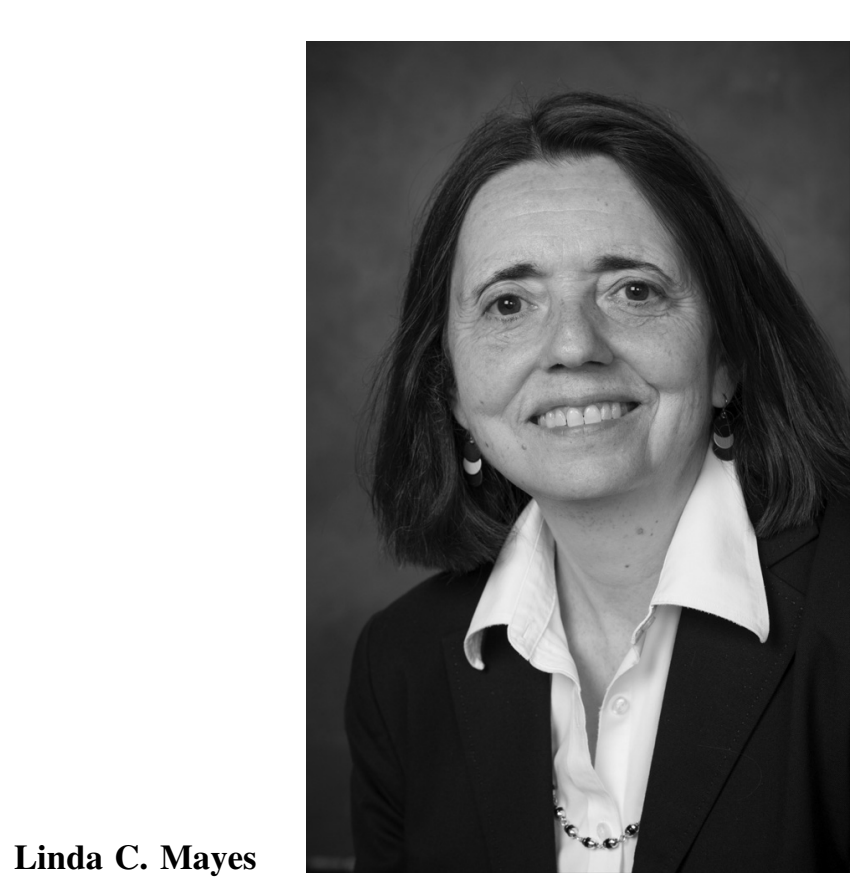

heightened emotion reactivity and overidentification of threat, possibly rendering the interpretation of children's socioaffective signals and calibration of stress responses more frustrating to caregivers whose mentalizing capacities may be already weakened because of preexisting adversities (Atzil et al., 2014; Fonagy \& Bateman, 2016). By the same token, struggling to empathize with the socioemotional needs of children due to maladaptive mentalizing in turn reduces caregiving satisfaction and increases caregiving stress, exacerbating risks for developing parental psychopathologies in families under chronic stress and adversities especially during the pandemic. Further, maladaptive mentalizing is prevalent in various forms of developmental psychopathology. Parents with a history of adversity exposure, such as those who experienced abuse and neglect as children, may struggle more to soothe their children and establish a secure attachment base at times of distress, both of which hinder children's ability to inspect and reflect on mental states of oneself and others in the long run (Luyten et al., 2020). The link between parental mentalizing and child psychopathology has also been validated using fMRI: internetwork connectivity important for mentalizing functions in the parental brain is prospectively associated with children's internalizing symptoms at age 6, and this association is mediated by children's emotion regulation skills (Abraham et al., 2018). In all, these findings highlight the dynamic influence of a chronic stressor like COVID-19 and other adversities on the parent-child dyad, suggesting novel considerations beyond individually-focused approaches in clinical settings.

\section{Emotional and Brain Synchrony of Parent-Child Dyads}

Another mechanism by which caregivers may regulate children's emotions is via emotional synchrony at the neural level. For example, children who exhibit a similar resting state functional connectome to their parents have more shared day-to-day emotional experiences with their parents (Lee et al., 2017). During cooperation, parents and children show brain synchrony in the PFC (dorsolateral PFC and frontopolar cortex; Reindl et al., 2018); and this synchrony is associated with children's daily emotional competence and regulation (Lee et al., 2017; Reindl et al., 2018). Importantly, a recent study found greater parenting stress to be associated with less parent/child brain synchrony in the PFC (e.g., inferior frontal gyrus, dorsolateral PFC) when subjects engaged in a dyadic task of watching animation videos together (Azhari et al., 2019). This study suggests that parent-child brain asynchrony may mediate the association between parenting stress and less dyadic coregulation (Azhari et al., 2019). Together, these studies underscore that parents/caregivers who are calm and regulated under stress (supported by PFC functions) may provide the behavioral and social signals that allow the child to regulate his or her own stress response or arousal (Feldman, 2015). Via multilevel interventions that aim to restore impacted PFC functions following the pandemic, the parent's and child's behavioral and neurobiological systems may become mutually attuned again, further setting the stage for children's emotional development.

Parents' experiences of heightened stress, like that which is occurring for nearly all parents during the COVID-19 pandemic (although much variability exists in the types and severity of pandemic stress), can result in maladaptive parenting behaviors. These can range from harsh over control or passive withdrawal to physical abuse or neglect. Parents' own experience with or risk of psychopathology also contributes to disruptions in sensitive caregiving and can exacerbate maladaptive reactions and behaviors in response to heightened stress in times of an unprecedented pandemic. For example, maternal depression is associated with greater disengagement from interactions with the child and more irritability and hostility toward the child (Lovejoy et al., 2000). Likewise, children whose parents suffer from substance abuse are at higher risks for child abuse and neglect (Nair et al., 2003).

With the idea that family-level factors play a central role in modulating caregivers' ability to buffer children's stress reactivity, recent evidence from the COVID-19 pandemic demonstrates that parenting stress and anxiety moderated the effect of pandemic-related stress on children's symptomatology (Cohodes et al., in press). That is, caregivers who reported higher levels of parenting stress and anxiety were less likely to effectively buffer children's stress during 


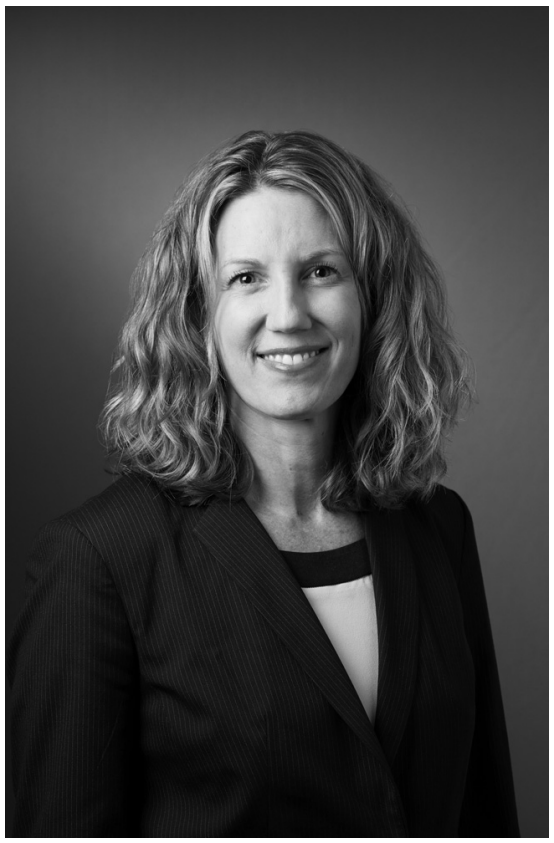

Carla S. Stover

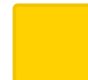

the COVID-19 pandemic (Cohodes et al., in press). By contrast, parents who engaged in higher levels of coaching of children's negative emotions and who maintained more stable home routines during the pandemic were more effectively able to buffer the effects of pandemic-related stress on children's symptomatology. These findings further highlight the central role that caregivers are playing in children's experiences of the pandemic, and the need to support caregivers in the context of heightened stress.

\section{System-, Family-, and Individual-Focused Interventions}

As described above, challenged caregiving and stress stemming from COVID-19 can significantly impact family functioning. Therefore, we can and should simultaneously prioritize interventions from the top-down-including at the systemic, family, and individual levels - to mitigate suboptimal mental and behavioral health outcomes for families at greatest risk during the pandemic.

To address systematic change, we advocate for the United States to prioritize policy changes that will help mitigate preexisting inequities and disadvantages, faced primarily by BIPOC (Black, Indigenous, and people of color) families, which, even before COVID-19, placed them at risk for suboptimal mental and behavioral health outcomes (Condon et al., 2020). Policies rooted in racism and discrimination place great burden on children and families of color with limited financial resources, limited access to quality education and health care, and individual, community, and historical-level trauma. The COVID-19 era has only exacerbated these inequities (Hooper et al., 2020; Van Dorn et al., 2020); placing such children and families at an even greater risk for psychopathologies. These children and families have been more likely to bear the burdens of 1) increased viral exposure (due to parents more likely to be low-wage front line workers without paid sick leave and difficulty with physical distancing in smaller homes; Oppel et al., 2020); 2) even more restricted resources (due to job insecurity for caregivers and adolescents, and increased food, housing, and health care insecurity; Power et al., 2020); 3) increased caregiver stress (due to forced choices between work and childcare, thereby putting caregivers at increased risk for anxiety, depression and substance abuse and children at increased risk for neglect, maltreatment, and family violence; Cluver et al., 2020; Humphreys et al., 2020); 4) the digital divide (due to lower-quality distance learning, both at the home and district levels, as well as reduced telehealth access and access to external support systems like teachers, counselors, and peers (Connecticut State Department of Education, 2020); and 5) increased inequities and bias (due to existing biases in health care and education; Oppel et al., 2020).

Policymakers must prioritize laws that tackle these social injustices, as this will not only help reduce inequities associated with toxic stress (World Health Organization [WHO] Commission on Social Determinants of Health, 2008; Taylor et al., 2016); but may also enable families to more effectively utilize the individual- and family-level interventions that will meet their immediate mental and behavioral health needs during the COVID-19 pandemic (see Figure 1). Such systemic interventions are broad in scope and include but are not limited to COVID-19 relief bills, a fair minimum wage, paid family/parental leave, expansion of Medicaid and/or universal health care, and equitable access to education including early childhood education and childcare. Improving equity in health care access is particularly key, including investment in a culturally and linguistically competent workforce, in ased availability of testing and personal protective eq . $_{\text {Y hent, }}$ and reduction of health care bias and stigma (fCondon et al., 2020). While it is beyond our scope to outline these policies in detail, many are evidence-based and known to reduce the risk of developing mental and behavioral health disorders (WHO Commission on Social Determinants of Health, 2008; Taylor et al., 2016).

While system-focused interventions are fundamental to mitigating the downstream effects of chronic stress, given the immediate needs of families during the COVID-19 pandemic, we advocate for the prioritization of structural and policy changes in concert with family- and individual-level interventions that cater to the specific needs of afflicted children and families. We strongly recommend investment in interventions that enable parents to provide optimal sensitive and supportive caregiving for their children during and 


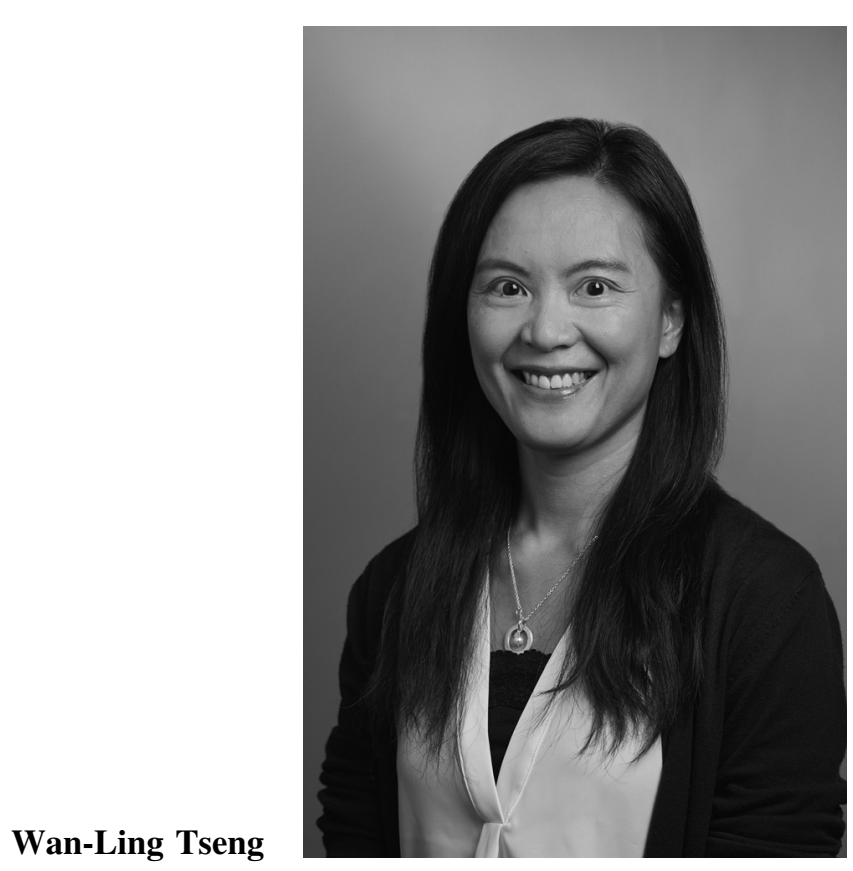

after the pandemic. As detailed in previous sections, uncontrollable stress caused by the pandemic impacts emotion regulation and mentalization skills. Interventions can focus on these mechanisms with parents individually, with caregivers and children simultaneously or in parallel sessions to target coregulation (Stob et al., 2020). Interventions that can help parents manage their stress and emotional overwhelm will aid them in better attending to the needs and stress of their children, especially at a time when parental stress level is at unprecedented levels. Parents' abilities to identify their own stress reactivity is critical to their ability to implement coping skills to manage their stress and ensure their PFC remains online for management of emotions, clear thinking and decision making. Treatments that focus on parents' reactivity and stress while building coping skills may be an immediate and initial approach to increase parents' capacities to attend to the stress and needs of their children during the pandemic.

There are multiple parenting interventions that target management of difficult child behaviors. Parent Management Treatments (PMTs) are evidence-based and widely implemented. Parents are often referred to these programs when they report difficulties in managing child behavior or when they come to the attention of social service agencies. Given this, these programs might be recommended during the pandemic. However, evidence suggests that PMTs are not effective at treating all families who seek services (McMahon \& Forehand, 2003) and are least effective with parents who are economically disadvantaged (Lundahl et al., 2006) and are underutilized by families at highest risk
(Kazdin \& Wassell, 1998). Meta-analysis of multiple studies of economically disadvantaged families found that mode of PMT intervention significantly moderated treatment outcomes with individually delivered modalities superior to group format (Lundahl et al., 2006).

Parental psychopathology has consistently been shown to moderate the effectiveness of PMTs (Hinshaw et al., 2015; McMahon \& Forehand, 2003). One reason for this may be that parents' heightened emotional reactivity and stress make it difficult for them to absorb the materials presented in PMTs or to apply them at home during stressful real life situations when they are unable to manage their own emotions. Several PMTs have been augmented to include modules or components to target the highest risk parents. Triple P (Sanders et al., 2004); the Incredible Years, and ParentChild Interaction Therapy (PCIT) have shown improved results with these enhancements (Gavita et al.2012; Maliken \& Katz, 2013). Notably, the dyadic psychosocial benefits and underlying neurobiology of PMTs have recently been validated in the context of Attachment Biobehavioral Catch-up (ABC), an evidence-based PMT. Valadez and colleagues (2020) demonstrated that high-risk infants of parents who received $\mathrm{ABC}$ showed fewer difficult behaviors in middle childhood, which was in turn mediated by greater functional activation to maternal cues in brain areas (such as the precuneus, cingulate gyrus, which have functional links to the PFC) important for social cognition (including mentalizing and processing of socioaffective signals) than children in the control intervention group. This intervention has been successfully implemented in some federally-funded school readiness programs, notably Early Head Start, which increases access to mental health support and educational opportunities for disadvantaged families in particular (Berlin et al., 2018).

There are also evidence-based treatments that target emotional reactivity and stress in adults. Dialectical behavior therapy (DBT) and treatments that use mindfulness stress reduction strategies can help parents with emotion regulation skills (Bogel et al., 2014; Lewallen \& Neece, 2015). DBT has been shown to improve suicidal and self-injurious behaviors for individuals with substantial difficulties with emotion dysregulation (Linehan, 1993; Neacsiu et al., 2014). DBT includes a skills training group to teach techniques to identify and label emotions, develop mindfulness strategies and build distress tolerance (Neacsiu et al., 2014; Valentine et al., 2015). Importantly, a small study of the impact of DBT treatment on parenting found that improvements in maternal emotion regulation benefited mothers' parenting (Martin et al., 2017). Selecting interventions that include a focus on parent emotion regulation first before considering parenting education or management skills may be the most helpful during the stress of the COVID-19 pandemic. 
Parents who are less able to attune to their own mental states and emotions will struggle to understand their children and help support them through emotion coaching. Mentalization-based treatments focus on helping parents mentalize for themselves and for their partners and children, and have been successful for families struggling with a host of adversities including parental substance misuse, domestic violence and child maltreatment (Stover et al., 2020; Suchman et al., 2010; Stob et al., 2020). Clinicians assist parents in understanding their own mental states and emotions and in how to use strategies to manage these emotions and stress to improve interactions with their children. These interventions also work with families through a trauma-focused lens, understanding how trauma and chronic stress impact the brain and functioning of both caregivers and their children. This makes these interventions a good match for atrisk families during COVID-19, in part because these treatments normalize emotions and include education about the body's stress response to aid parents in learning to manage emotions and teach those skills to their children.

Dyadic interventions like Child-Parent Psychotherapy (Lieberman et al., 2005) and Minding the Baby (Sadler et al., 2006) work from an attachment framework to improve parent mentalizing, thereby increasing the child's feelings of supportive and responsive caregiving, promoting secure attachment, and improving functioning of both parent and child (Ippen et al., 2011; Lieberman et al., 2005). Other evidence-based interventions like Mothering from the Inside Out (Suchman et al., 2010) and Fathers for Change (Stover et al., 2020) work with parents individually to address similar goals. They focus first on helping at-risk parents with histories of substance misuse and intimate partner violence (IPV) mentalize for themselves and manage their own emotions and then help parents focus on the thoughts, experience and emotions of their children. Fathers for Change, for example, helps fathers with histories of use of family violence and substance misuse identify their feelings of stress and triggers for emotion dysregulation. This is followed by teaching fathers about their stress response system and how breathing and grounding techniques can put them back in charge of their physiology, emotions and behaviors (Stover et al., 2020). And, a new intervention technique called The Family Cycle relies on mentalizing skills to break the intergenerational transmission of trauma (Stob et al., 2020). A focus on mentalizing and emotion regulation is key during times of chronic stress such as during the COVID-19 pandemic and its aftermath given potential for overload of the stress system, compromising PFC function and increasing risk for IPV and child maltreatment.

Several other evidence-based interventions that specifically address acute stress and posttraumatic stress disorder (PTSD) take a family-focused approach to increase parent support of children during times of stress and trauma. These interventions are appropriate for children who have experienced a traumatic event and have trauma-related symptoms and impairment, either due to trauma experienced during the pandemic (e.g., medical trauma, witnessing, experiencing family violence, sudden loss of a family member or fear of loss of a family member), or by pandemic related triggers that exacerbate earlier life traumatic events (Bartlett et al., 2020; Bryant et al., 2020). Trauma Focused-Cognitive Behavioral Therapy (Mannarino et al., 2014) includes parallel parent sessions and conjoint parent-child sessions to help parents provide support to their child, thereby creating the supportive environment the child needs to recover. The Child and Family Traumatic Stress Intervention (Marans et al., 2014) includes a focus on parent-child sessions to help parents support their children in recovery posttrauma. Both of these interventions teach coping strategies similar to those described above related to the body's stress response. These strategies mitigate traumatic stress responses following trauma and are helpful for mitigating ongoing stress. Although focused on the child's symptoms, these interventions consider the parents' symptoms and functioning and teach coping skills to both parent and child, allowing parents to manage their own stress reactivities and emotion regulation difficulties while also supporting their child.

Overall, treatments that focus on the stress response and improved mentalization and emotion regulation are likely to have the most significant impact at the individual and family level, regardless of whether one of the named interventions above is used. Providers should incorporate a focus on these areas into their interventions with families during COVID-19. However, of significant concern is whether families can access the described practices due to lack of availability or access in their communities. Many of the interventions described have been implemented via telehealth since agencies moved away from in-person treatment during state lockdowns. This delivery removed some barriers to access in the wake of COVID-19; however, lack of health insurance coverage, access to reliable technology and Internet access can all prevent access even when the service is available remotely, as can other barriers like a lack of providers who can provide services in languages other than English. We emphasize again that these issues require simultaneous systematic changes (see Figure 1) to ensure the creativity shown by providers during this pandemic can reach all who need it.

Agencies and intervention developers rapidly offered training for telehealth providers and modified strategies that were once less possible virtually. Other creative ways such as mailing session materials to families to use during virtual sessions, restructuring sessions to provide privacy at home, and screen sharing of information have all seen success, but may not be widely available to the most vulnerable families that fundamentally lack access to these telehealth and Internet services. The discrepancy between service innovation and its actual implementation alongside 
Figure 1

A Multilevel, Neuroscience-Informed Approach to Preventing and Treating Poor Mental Health Related to the COVID-19 Pandemic

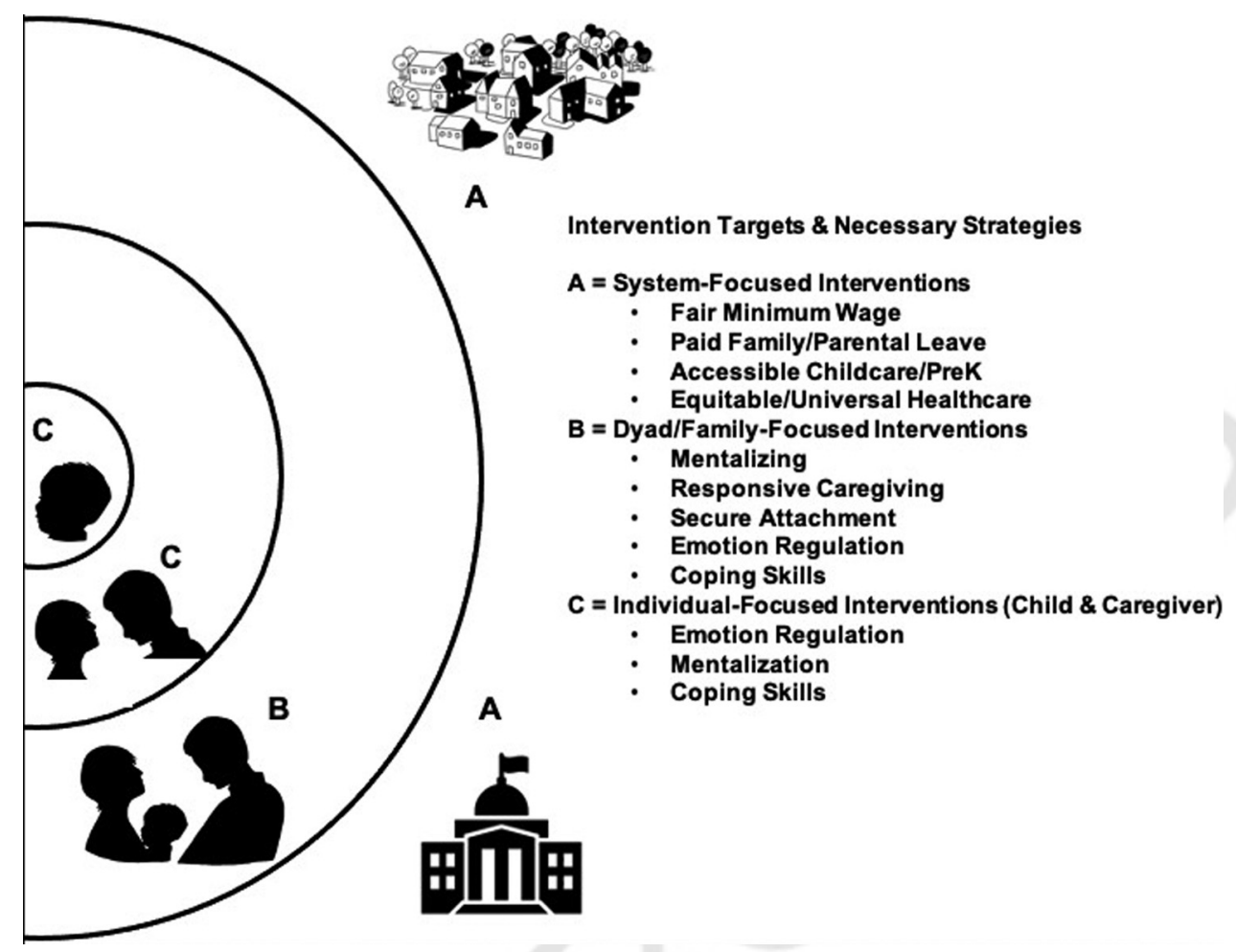

Note. System-focused interventions (A) that address and dismantle historically racist policies are needed to reduce long-standing inequities that were exacerbated by the COVID-19 pandemic. System-focused interventions will also provide foundational support to enable families to access and utilize family (B) and individual (C) focused interventions.

preexisting disparities supports the foundational idea that multilevel interventions should be taken in concert so as to achieve optimal and sustainable efficacy. Family- and individual-level interventions are best implemented only when there is a collective goal to simultaneously mitigate the systemic inequities that disproportionately harm the mental health of marginalized families.

\section{Conclusion}

The COVID-19 pandemic is both an acute and chronic stressor that has exacerbated preexisting inequities and resulting risks for mental and behavioral health disorders, particularly for disadvantaged and marginalized children and families. These inequities are rooted in systemic racism and long-standing racist policies. The heightened duress resulting from the COVID-19 pandemic places these vulnerable community members at increased risk for chronic stress, which can disrupt brain functioning associated with heightened emotional reactivity, emotion dysregulation, blunted reward sensitivity, and maladaptive mentalizing. The deleterious effects of chronic stress and its underlying neurobiology can be buffered by sensitive caregiving, which can be supported and enhanced by evidence-based interventions given to children, parents, and families. However, these interventions cannot succeed without the simultaneous prioritization of policies that dismantle the inequities resulting from systemic racism, which will reduce family stress and enable families to access the interventions they need and deserve.

\section{References}

Abraham, E., Raz, G., Zagoory-Sharon, O., \& Feldman, R. (2018). Empathy networks in the parental brain and their long-term effects on children's stress reactivity and behavior adaptation. Neuropsychologia, 116(Pt A), 75-85. https://doi.org/10.1016/j.neuropsychologia.2017.04 .015

Adams, E. L., Caccavale, L. J., Smith, D., \& Bean, M. K. (2020). Food insecurity, the home food environment, and parent feeding practices in the era of COVID-19. Obesity, 28(11), 2056-2063. https://doi.org/10 $.1002 /$ oby. 22996

Ameriean Psychologieal Association. (2020). Stress in the Time of COVH-19. Stress in Ameriea 2020 Survey. Retrieved from https.th Wapa.orghewstpress/releases/stress/2020/report

Arnsten, A. F. T. (2009). Stress signalling pathways that impair prefrontal cortex structure and function. Nature Reviews Neuroscience, 10(6), 410-422. https://doi.org/10.1038/nrn2648 
Arnsten, A. F. T. (2015). Stress weakens prefrontal networks: Molecular insults to higher cognition. Nature Neuroscience, 18(10), 1376-1385. https://doi.org/10.1038/nn.4087

Atzil, S., Hendler, T., \& Feldman, R. (2014). The brain basis of social synchrony. Social Cognitive and Affective Neuroscience, 9(8), 1193-1202. https://doi.org/10.1093/scan/nst105

Azhari, A., Leck, W. Q., Gabrieli, G., Bizzego, A., Rigo, P., Setoh, P., Bornstein, M. H., \& Esposito, G. (2019). Parenting stress undermines mother-child brain-to-brain synchrony: A hyperscanning study. Scientific Reports, 9(1), 1-9. https://doi.org/10.1038/s41598-019-47810-4

Bartlett, J. D., Griffin, J., \& Thomson, D. (2020). Resources for supporting children's emotional well-being during the COVID-19 pandemic. Child Trends. Retrieved from https://escholarship.umassmed.edu/covid19/5

Berlin, L. J., Martoccio, T. L., \& Jones Harden, B. (2018). Improving Early Head Start impacts on parenting through attachment-based intervention: A randomized controlled trial. Developmental Psychology, 54(12), 2316-2327. https://doi.org/10.1037/dev0000592

Bloss, E. B., Janssen, W. G., Ohm, D. T., Yuk, F. J., Wadsworth, S., Saardi, K. M., McEwen, B. S., \& Morrison, J. H. (2011). Evidence for reduced experience-dependent dendritic spine plasticity in the aging prefrontal cortex. The Journal of Neuroscience: The Official Journal of the Society for Neuroscience, 31(21), 7831-7839. https://doi.org/10.1523/ JNEUROSCI.0839-11.2011

Blum, K., Braverman, E. R., Holder, J. M., Lubar, J. F., Monastra, V. J., Miller, D., Lubar, J. O., Chen, T. J. H., \& Comings, D. E. (2000). The reward deficiency syndrome: A biogenetic model for the diagnosis and treatment of impulsive, addictive and compulsive behaviors. Journal of Psychoactive Drugs, 32(sup1), 1-112. https://doi.org/10.1080/02791072 2000.10736099

Breslau, N. (2009). The epidemiology of trauma, PTSD, and other posttrauma disorders. Trauma, Violence \& Abuse, 10(3), 198-210. https:// doi.org/10.1177/1524838009334448

Briggs-Gowan, M. J., Pollak, S. D., Grasso, D., Voss, J., Mian, N. D., Zobel, E., McCarthy, K. J., Wakschlag, L. S., \& Pine, D. S. (2015). Attention bias and anxiety in young children exposed to family violence. Journal of Child Psychology and Psychiatry, and Allied Disciplines, 56(11), 1194-1201. https://doi.org/10.1111/jcpp.12397

Brown, S. M., Doom, J. R., Lechuga-Peña, S., Watamura, S. E., \& Koppels, T. (2020). Stress and parenting during the global COVID-19 pandemic. Child Abuse \& Neglect: The International Journal, 110, 104699. Advance online publication. https://doi.org/10.1016/j.chiabu .2020 .104699

Bryant, D. J., Oo, M., \& Damian, A. J. (2020). The rise of adverse childhood experiences during the COVID-19 pandemic. Psychological Trauma: Theory, Research, Practice, and Policy, 12(S1), S193-S194. https://doi.org/10.1037/tra0000711

Cai, Y., Kim, D. J., Takahashi, T., Broadhurst, D. I., Ma, S., Rattray, N. J., . Mao, T. (2020). Kynurenic acid underlies sex-specific immune responses to COVID-19. medRxiv. Advance online publication. https:// doi.org/10.1101/2020.09.06.20189159

Callaghan, B., Meyer, H., Opendak, M., Van Tieghem, M., Harmon, C., Li, A., Lee, F. S., Sullivan, R. M., \& Tottenham, N. (2019). Using a developmental ecology framework to align fear neurobiology across species. Annual Review of Clinical Psychology, 15(1), 345-369. https://doi .org/10.1146/annurev-clinpsy-050718-095727

Carpenter, A. L., Elkins, R. M., Kerns, C., Chou, T., Greif Green, J., \& Comer, J. S. (2017). Event-related household discussions following the Boston Marathon bombing and associated posttraumatic stress among area youth. Journal of Clinical Child and Adolescent Psychology, 46(3), 331-342. https://doi.org/10.1080/15374416.2015.1063432

Cluver, L., Lachman, J. M., Sherr, L., Wessels, I., Krug, E., Rakotomalala, S., Blight, S., Hillis, S., Bachman, G., Green, O., Butchart, A., Tomlinson, M., Ward, C. L., Doubt, J., \& McDonald, K. (2020).
Parenting in a time of COVID-19. Lancet, 395(10231), Article e64 https://doi.org/10.1016/S0140-6736(20)30736-4

Cohodes, E. M., Kitt, E. R., Baskin-Sommers, A., \& Gee, D. G. (2020) Influences of early-life stress on frontolimbic circuitry: Harnessing : dimensional approach to elucidate the effects of heterogeneity in stress exposure. Developmental Psychobiology. Advance Online Publication. https://doi.org/10.1002/dev.21969

Cohodes, E. M., McCauley, S., \& Gee, D. G., (in press). Parental buffering of stress in the time of COVID-19: Family level factors may moderate the association between pandemic-related stress and youth symptomatology. Journal of Abnormal Child Psychology.

Condon, E. M., Dettmer, A. M., Gee, D. G., Hagan, C., Lee, K. S., Mayes, L. C., Stover, C. S., \& Tseng, W-L. (2020). Commentary: COVID-19 and mental health equity in the United States. Froniters in Sociology, https://doi.org/10.3389/fsoc.2020.584390

Connecticut State Department of Education. (2020). Student participation in distance learning: device/connectivity needs, effective strategies, challenges, and state supports needed. Retrieved from https://portal.ct .gov/-/media/SDE/Performance/Research-Library/Results-from-DistrictSurvey-on-Student-Participation-in-Distance-Learning-and-DeviceConnectivity-N.pdf

Dennison, M. J., Rosen, M. L., Sambrook, K. A., Jenness, J. L., Sheridan, M. A., \& McLaughlin, K. A. (2019). Differential associations of distinct forms of childhood adversity with neurobehavioral measures of reward processing: A developmental pathway to depression. Child Development, 90(1), e96-e113. https://doi.org/10.1111/cdev.13011

Duffy, K. A., Mclaughlin, K. A., \& Green, P. A. (2018). Early life adversity and health-risk behaviors: Proposed psychological and neural mechanisms. Annals of the New York Academy of Sciences, 1428(1), 151-169. https://doi.org/10.1111/nyas. 13928

Feldman, R. (2015). The adaptive human parental brain: Implications for children's social development. Trends in Neurosciences, 38(6), 387-399. https://doi.org/10.1016/j.tins.2015.04.004

Fonagy, P., \& Bateman, A. W. (2016). Adversity, attachment, and mentalizing. Comprehensive Psychiatry, 64(?), 59-66. https://doi.org/10.1016/j .comppsych.2015.11.006

Gee, D. G., \& Casey, B. J. (2015). The impact of developmental timing for stress and recovery. Neurobiology of Stress, 1(?),184-194. https://doi .org/10.1016/j.ynstr.2015.02.001

Gee, D. G., Humphreys, K. L., Flannery, J., Goff, B., Telzer, E. H., Shapiro, M., Hare, T. A., Bookheimer, S. Y., \& Tottenham, N. (2013). A developmental shift from positive to negative connectivity in human amygdala-prefrontal circuitry. The Journal of Neuroscience: The Official Journal of the Society for Neuroscience, 33(10), 4584-4593. https:// doi.org/10.1523/JNEUROSCI.3446-12.2013

Germine, L., Dunn, E. C., McLaughlin, K. A., \& Smoller, J. W. (2015). Childhood adversity is associated with adult theory of mind and social affiliation, but not face processing. PLoS ONE, 10(6).

Hains, A. B., Vu, M. A. T., Maciejewski, P. K., Van Dyck, C. H., Gottron, M., \& Arnsten, A. F. T. (2009). Inhibition of protein kinase C signaling protects prefrontal cortex dendritic spines and cognition from the effects of chronic stress. Proceedings of the National Academy of Sciences, USA, USA of the United States of America, 106(42), 17957-17962. https://doi.org/10.1073/pnas.0908563106

Hanson, J. L., Hariri, A. R., \& Williamson, D. E. (2015). Blunted ventral striatum development in adolescence reflects emotional neglect and predicts depressive symptoms. Biological Psychiatry, 78(9), 598-605. https://doi.org/10.1016/j.biopsych.2015.05.010

Hein, T. C., \& Monk, C. S. (2017). Research Review: Neural response to threat in children, adolescents, and adults after child maltreatment-A quantitative meta-analysis. Journal of Child Psychology and Psychiatry, and Allied Disciplines, 58(3), 222-230. https://doi.org/10.1111/jcpp .12651 
Heleniak, C., Jenness, J. L., Vander Stoep, A., McCauley, E., \& McLaughlin, K. A. (2016). Childhood maltreatment exposure and disruptions in emotion regulation: A transdiagnostic pathway to adolescent internalizing and externalizing psychopathology. Cognitive Therapy and Research, 40(3), 394-415. https://doi.org/10.1007/s10608-015-9735-Z

Hinshaw, S., Arnold, L. E., \& The MTA Cooperative Group. (2015). ADHD, multimodal treatment, and longitudinal outcome: Evidence, paradox, and challenge. Wiley Interdisciplinary Reviews: Cognitive Science, 6(1), 39-52. https://doi.org/10.1002/wcs.1324

Hooper, M. W., Nápoles, A. M., \& Pérez-Stable, E. J. (2020). COVID-19 and racial/ethnic disparities. Journal of the American Medical Association, 323(24), 2466-2467. https://doi.org/10.1001/jama.2020.8598

Humphreys, K. L., Myint, M. T., \& Zeanah, C. H. (2020). Increased risk for family violence during the COVID-19 pandemic. Pediatrics, 145(4), Article e20200982. https://doi.org/10.1542/peds.2020-0982

Ippen, C. G., Harris, W. W., Van Horn, P., \& Lieberman, A. F. (2011). Traumatic and stressful events in early childhood: Can treatment help those at highest risk? Child Abuse \& Neglect: The International Journal, 35(7), 504-513. https://doi.org/10.1016/j.chiabu.2011.03.009

Kazdin, A. E., \& Wassell, G. (1998). Treatment completion and therapeutic change among children referred for outpatient therapy. Professional Psychology, Research and Practice, 29(4), 332-340. https://doi.org/10 $.1037 / 0735-7028.29 .4 .332$

Kertes, D. A., Donzella, B., Talge, N. M., Garvin, M. C., Van Ryzin, M. J., \& Gunnar, M. R. (2009). Inhibited temperament and parent emotional availability differentially predict young children's cortisol responses to novel social and nonsocial events. Developmental Psychobiology, 51(7), 521-532. https://doi.org/10.1002/dev.20390

Kim, J., \& Cicchetti, D. (2010). Longitudinal pathways linking child maltreatment, emotion regulation, peer relations, and psychopathology. Journal of Child Psychology and Psychiatry, and Allied Disciplines, 51(6), 706-716. https://doi.org/10.1111/j.1469-7610.2009.02202.x

Kim, P., Evans, G. W., Angstadt, M., Ho, S. S., Sripada, C. S., Swain, J. E., Liberzon, I., \& Phan, K. L. (2013). Effects of childhood poverty and chronic stress on emotion regulatory brain function in adulthood. Proceedings of the National Academy of Sciences, USA, USA of the United States of America, 110(46), 18442-18447. https://doi.org/10 $.1073 /$ pnas. 1308240110

Landolt, M. A., Ystrom, E., Sennhauser, F. I., Gnehm, H. E., \& Volltath, M. E. (2012). The muttual prospeetive influienee of ehitd and parental post-traumatic stress symptoms in pediatrie patients. Journal of Chitd Psychotogy and Psychiatry, and Altied Disciptines, 53(7), 767-774.

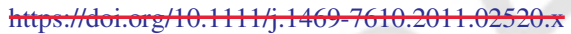

Lee, T. H., Miernicki, M. E., \& Telzer, E. H. (2017). Families that fire together smile together: Resting state connectome similarity and daily emotional synchrony in parent-child dyads. NeuroImage, 152(?), 31-37. https://doi.org/10.1016/j.neuroimage.2017.02.078

Levitt, P., \& Eagleson, K. L. (2018). The ingredients of healthy brain and child development. Washington University Journal of Law and Policy, 57(?), 75. Retrieved from https://openscholarship.wustl.edu/law_journal _law_policy/vol57/iss1/9

Lewallen, A. C., \& Neece, C. L. (2015). Improved social skills in children with developmental delays after parent participation in MBSR: The role of parent-child relational factors. Journal of Child and Family Studies, 24(10), 3117-3129. https://doi.org/10.1007/s10826-015-0116-8

Lieberman, A. F., Van Horn, P., \& Ippen, C. G. (2005). Toward evidencebased treatment: Child-parent psychotherapy with preschoolers exposed to marital violence. Journal of the American Academy of Child \& Adolescent Psychiatry, 44(12), 1241-1248. https://doi.org/10.1097/01.chi .0000181047 .59702 .58

Linehan, M. M. (1993). Cognitive behavioral treatment of borderline personality disorder. Guilford Press Press.

Liston, C., McEwen, B. S., \& Casey, B. J. (2009). Psychosocial stress reversibly disrupts prefrontal processing and attentional control.
Proceedings of the National Academy of Sciences, USA, USA of the United States of America, 106(3), 912-917. https://doi.org/10.1073/ pnas.0807041106

Liston, C., Miller, M. M., Goldwater, D. S., Radley, J. J., Rocher, A. B., Hof, P. R., Morrison, J. H., \& McEwen, B. S. (2006). Stress-induced alterations in prefrontal cortical dendritic morphology predict selective impairments in perceptual attentional set-shifting. The Journal of Neuroscience: The Official Journal of the Society for Neuroscience, 26(30), 7870-7874. https://doi.org/10.1523/JNEUROSCI.1184-06.2006

Loades, M. E., Chatburn, E., Higson-Sweeney, N., Reynolds, S., Shafran, R., Brigden, A., Linney, C., McManus, M. N., Borwick, C., \& Crawley, E. (2020). Rapid systematic review: The impact of social isolation and loneliness on the mental health of children and adolescents in the context of COVID-19. Journal of the American Academy of Child \& Adolescent Psychiatry, 59(11), 1218-1239. https://doi.org/10.1016/j.jaac .2020 .05 .009

Lovejoy, M. C., Graczyk, P. A., O'Hare, E., \& Neuman, G. (2000). Maternal depression and parenting behavior: A meta-analytic review. Clinical Psychology Review, 20(5), 561-592. https://doi.org/10.1016/S0272 -7358(98)00100-7

Lundahl, B., Risser, H. J., \& Lovejoy, M. C. (2006). A meta-analysis of parent training: Moderators and follow-up effects. Clinical Psychology Review, 26(1), 86-104. https://doi.org/10.1016/j.cpr.2005.07.004

Lupien, S. J., Parent, S., Evans, A. C., Tremblay, R. E., Zelazo, P. D., Corbo, V., Pruessner, J. C., \& Séguin, J. R. (2011). Larger amygdala but no change in hippocampal volume in 10-year-old children exposed to maternal depressive symptomatology since birth. Proceedings of the National Academy of Sciences, USA, USA of the United States of America, 108(34), 14324-14329. https://doi.org/10.1073/pnas.1105371108

Luyten, P., Campbell, C., Allison, E., \& Fonagy, P. (2020). The mentalizing approach to psychopathology: State of the art and future directions. Annual Review of Clinical Psychology, 16, 297-325. https://doi.org/10 $.1146 /$ annurev-clinpsy-071919-015355

Maliken, A. C., \& Katz, L. F. (2013). Exploring the impact of parental psychopathology and emotion regulation on evidence-based parenting interventions: A transdiagnostic approach to improving treatment effectiveness. Clinical Child and Family Psychology Review, 16(2), 173-186. https://doi.org/10.1007/s10567-013-0132-4

Mannarino, A. P., Cohen, J. A., \& Deblinger, E. (2014). Trauma-focused cognitive-behavioral therapy (pp. 165-185). https://doi.org/10.1007/978 -94-007-7404-9_10

Marans, S., Epstein, C., \& Berkowitz, S. (2014). The child and family traumatic stress intervention treatment application for young children. Childhood Violent Trauma Center.

Martin, C. G., Roos, L. E., Zalewski, M., \& Cummins, N. (2017). A dialectical behavior therapy skills group case study on mothers with severe emotion dysregulation. Cognitive and Behavioral Practice, 24(4), 405-415. https://doi.org/10.1016/j.cbpra.2016.08.002

McEwen, C. A., \& McEwen, B. S. (2017). Social structure, adversity, toxic stress, and intergenerational poverty: An early childhood model. Аnпиаl Review of Sociology, 43(1), 445-472. https://doi.org/10.1146/annurevsoc-060116-053252

McLaughlin, K. A., Conron, K. J., Koenen, K. C., \& Gilman, S. E. (2010). Childhood adversity, adult stressful life events, and risk of past-year psychiatric disorder: A test of the stress sensitization hypothesis in a population-based sample of adults. Psychological Medicine, 40(10), 1647-1658. https://doi.org/10.1017/S0033291709992121

McMahon, R. J., \& Forehand, R. (2003). Helping the noncompliant child: A clinician's guide to effective parent training. Guilford Press.

Moriceau, S., \& Sullivan, R. M. (2006). Maternal presence serves as a switch between learning fear and attraction in infancy. Nature Neuroscience, 9(8), 1004-1006. https://doi.org/10.1038/nn1733

Nair, P., Schuler, M. E., Black, M. M., Kettinger, L., \& Harrington, D. (2003). Cumulative environmental risk in substance abusing women: 
Early intervention, parenting stress, child abuse potential and child development. Child Abuse \& Neglect: The International Journal, 27(9), 997-1017. https://doi.org/10.1016/S0145-2134(03)00169-8

Neacsiu, A. D., Eberle, J. W., Kramer, R., Wiesmann, T., \& Linehan, M. M. (2014). Dialectical behavior therapy skills for transdiagnostic emotion dysregulation: A pilot randomized controlled trial. Behaviour Research and Therapy, 59, 40-51. https://doi.org/10.1016/j.brat.2014.05 .005

Opendak, M., Robinson-Drummer, P., Blomkvist, A., Zanca, R. M., Wood, K., Jacobs, L., Chan, S., Tan, S., Woo, J., Venkataraman, G., Kirschner, E., Lundström, J. N., Wilson, D. A., Serrano, P. A., \& Sullivan, R. M. (2019). Neurobiology of maternal regulation of infant fear: The role of mesolimbic dopamine and its disruption by maltreatment. Neuropsychopharmacology, 44(7), 1247-1257. https://doi.org/10 1038/s41386-019-0340-9

Oppel, R. A., Gebeloff, R., Lai, K. K. R., Wright, W., \& Smith, M. (2020, July 5) The fullest look yet at the racial inequity of coronavirus. The New York Times. Retrieved from https://www.nytimes.com/ interactive/2020/07/05/us/coronavirus-latinos-african-americans-cdcdata.html

Orben, A., Tomova, L., \& Blakemore, S. J. (2020). The effects of social deprivation on adolescent development and mental health. The Lancet. Child \& Adolescent Health, 4(8), 634-640. https://doi.org/10.1016/ S2352-4642(20)30186-3

Ota, K. T., Liu, R.-J., Voleti, B., Maldonado-Aviles, J. G., Duric, V., Iwata, M., Dutheil, S., Duman, C., Boikess, S., Lewis, D. A., Stockmeier, C. A., DiLeone, R. J., Rex, C., Aghajanian, G. K., \& Duman, R. S. (2014). REDD1 is essential for stress-induced synaptic loss and depressive behavior. Nature Medicine, 20(5), 531-535. https:// doi.org/10.1038/nm.3513

Palmer, F. B., Anand, K. J. S., Graff, J. C., Murphy, L. E., Qu, Y., Völgyi, E., Rovnaghi, C. R., Moore, A., Tran, Q. T., \& Tylavsky, F. A. (2013). Early adversity, socioemotional development, and stress in urban 1year-old children. The Journal of Pediatrics, 163(6), 1733-1739.e1. https://doi.org/10.1016/j.jpeds.2013.08.030

Park, A. T., Leonard, J. A., Saxler, P. K., Cyr, A. B., Gabrieli, J. D. E., \& Mackey, A. P. (2018). Amygdala-medial prefrontal cortex connectivity relates to stress and mental health in early childhood. Social Cognitive and Affective Neuroscience, 13(4), 430-439. https://doi.org/10.1093/ scan/nsy017

Patrick, S. W., Henkhaus, L. E., Zickafoose, J. S., Lovell, K., Halvorson, A., Loch, S., Letterie, M., \& Davis, M. M. (2020). Well-being of parents and children during the COVID-19 pandemic: A national survey. Pediatrics, 146(4), Article e2020016824. Advance online publication. https:// doi.org/10.1542/peds.2020-016824

Pollak, S. D., \& Tolley-Schell, S. A. (2003). Selective attention to facial emotion in physically abused children. Journal of Abnormal Psychology, 112(3), 323-338. https://doi.org/10.1037/0021-843X.112.3.323

Power, M., Doherty, B., Pybus, K., \& Pickett, K. (2020). How COVID-19 has exposed inequalities in the U.K. food system: The case of U.K. food and poverty. Emerald Open Research, 2(?), 11. https://doi.org/10 .35241/emeraldopenres.13539.1

Reindl, V., Gerloff, C., Scharke, W., \& Konrad, K. (2018). Brain-to-brain synchrony in parent-child dyads and the relationship with emotion regulation revealed by fNIRS-based hyperscanning. NeuroImage, 178(?), 493-502. https://doi.org/10.1016/j.neuroimage.2018.05.060

Rundle, A. G., Park, Y., Herbstman, J. B., Kinsey, E. W., \& Wang, Y. C. (2020). COVID-19-related school closings and risk of weight gain among children. Obesity, 28(6), 1008-1009. https://doi.org/10.1002/oby .22813

Sanchez, M. M. (2006). The impact of early adverse care on HPA axis development: Nonhuman primate models. Hormones and Behavior, 50(4), 623-631. https://doi.org/10.1016/j.yhbeh.2006.06.012
Sanchez, M. M., McCormack, K. M., \& Howell, B. R. (2015). Social buffering of stress responses in nonhuman primates: Maternal regulation of the development of emotional regulatory brain circuits. Social Neuroscience, 10(5), 512-526. https://doi.org/10.1080/17470919.2015 .1087426

Sanders, M. R., Pidgeon, A. M., Gravestock, F., Connors, M. D., Brown, S., \& Young, R. W. (2004). Does parental attributional retraining and anger management enhance the effects of the Triple PPositive Parenting Program with parents at risk of child maltreatment? Behavior Therapy, 35(3), 513-535. https://doi.org/10.1016/ S0005-7894(04)80030-3

Shipman, K. L., Schneider, R., Fitzgerald, M. M., Sims, C., Swisher, L., \& Edwards, A. (2007). Maternal emotion socialization in maltreating and non-maltreating families: Implications for children's emotion regulation. Social Development, 16(2), 268-285. https://doi.org/10.1111/j.14679507.2007.00384.x

Silvers, J. A., Insel, C., Powers, A., Franz, P., Helion, C., Martin, R. E. Weber, J., Mischel, W., Casey, B. J., \& Ochsner, K. N. (2017). vlPFC-vmPFC-amygdala interactions underlie age-related differences in cognitive regulation of emotion. Cerebral Cortex (New York, N.Y, ), 27(7), 3502-3514. https://doi.org/10.1093/cercor/bhw073

Stob, V., Slade, A., Adnopoz, J., \& Woolston, J. (2020). The family cycle: Breaking the intergenerational transmission of trauma through mentalizing. Journal of Infant, Child, and Adolescent Psychotherapy, JCAP, 19(?),1-16. https://doi.org/10.1080/15289168.2020.1786762

Stover, C. S., Beebe, R., Clough, M., DiVietro, S., Madigan, L., \& Grasso, D. (2020). Statewide implementation of Fathers for Change within child welfare: A fathering intervention for families impacted by partner violence. Journal of Family Violence. Advance online publication. https:// doi.org/10.1007/s10896-020-00199-5

Suchman, N. E., DeCoste, C., Castiglioni, N., McMahon, T. J., Rounsaville, B., \& Mayes, L. (2010). The Mothers and Toddlers Program, an attachment-based parenting intervention for substance using women: Post-treatment results from a randomized clinical pilot. Attachment \& Human Development, 12(5), 483-504. https://doi.org/10.1080/ 14616734.2010.501983

Taylor, L. A., Tan, A. X., Coyle, C. E., Ndumele, C., Rogan, E., Canavan, M., Curry, L. A., \& Bradley, E. H. (2016). Leveraging the social determinants of health: What works? PLOS ONE, 11(8), Article e0160217. https://doi.org/10.1371/journal.pone.0160217

United Nations. (2020). Policy brief: The impact of COVID-19 on children. Retrieved from https://unsdg.un.org/resources/policy-briefimpact-covid-19-children

Valadez, E. A., Tottenham, N., Tabachnick, A. R., \& Dozier, M. (2020). Early parenting intervention effects on brain responses to maternal cues among high-risk children. American Journal of Psychiatry, 177(9), 818-826. https://doi.org/10.1176/appi.ajp.2020.20010011

Valentine, S. E., Bankoff, S. M., Poulin, R. M., Reidler, E. B., \& Pantalone, D. W. (2015). The use of dialectical behavior therapy skills training as stand-alone treatment: A systematic review of the treatment outcome literature. Journal of Clinical Psychology, 71(1), 1-20. https:// doi.org/10.1002/jclp.22114

Van Dorn, A., Cooney, R. E., \& Sabin, M. L. (2020). COVID-19 exacerbating inequalities in the U.S Lancet, 395(10232), 1243-1244. https:// doi.org/10.1016/S0140-6736(20)30893-X

Verdery, A. M., Smith-Greenaway, E., Margolis, R., \& Daw, J. (2020). Tracking the reach of COVID-19 kin loss with a bereavement multiplier applied to the United States. Proceedings of the National Academy of Sciences, USA, USA of the United States of America, 117(30), 17695-17701. https://doi.org/10.1073/pnas.2007476117

Vyas, A., Mitra, R., Shankaranarayana Rao, B. S., \& Chattarji, S. (2002). Chronic stress induces contrasting patterns of dendritic remodeling in hippocampal and amygdaloid neurons. The Journal of Neuroscience: 
The Official Journal of the Society for Neuroscience, 22(15), 6810-6818. https://doi.org/10.1523/JNEUROSCI.22-15-06810.2002

Whittle, S., Dennison, M., Vijayakumar, N., Simmons, J. G., Yücel, M., Lubman, D. I., Pantelis, C., \& Allen, N. B. (2013). Childhood maltreatment and psychopathology affect brain development during adolescence. Journal of the American Academy of Child \& Adolescent Psychiatry, 52(9), 940-952. https://doi.org/10.1016/j.jaac.2013 .06 .007

WHO Commission on Social Determinants of Health. (2008). Closing the gap in a generation: Health equity through action on the social determinants of health: Commission on social determinants of health final report. Retrieved from https://www.who.int/social_determinants/ thecommission/finalreport/en/
Received August 31, 2020

Revision received January 7, 2021

Accepted January 14, 2021 